The Annals of Statistic

2015, Vol. 43, No. 2, 878-902

DOI: $10.1214 / 14-\mathrm{AOS} 1304$

(C) Institute of Mathematical Statistics, 2015

\title{
ASYMPTOTICALLY DISTRIBUTION-FREE GOODNESS-OF-FIT TESTING FOR TAIL COPULAS
}

\author{
By Sami Umut Can ${ }^{*}, 1$, John H. J. EinmahL ${ }^{\dagger}$, \\ Estate V. Khmaladze ${ }^{\ddagger}$ And Roger J. A. Laeven* \\ University of Amsterdam* ${ }^{*}$, Tilburg University ${ }^{\dagger}$ \\ and Victoria University of Wellington ${ }^{\ddagger}$
}

\begin{abstract}
Let $\left(X_{1}, Y_{1}\right), \ldots,\left(X_{n}, Y_{n}\right)$ be an i.i.d. sample from a bivariate distribution function that lies in the max-domain of attraction of an extreme value distribution. The asymptotic joint distribution of the standardized component-wise maxima $\bigvee_{i=1}^{n} X_{i}$ and $\bigvee_{i=1}^{n} Y_{i}$ is then characterized by the marginal extreme value indices and the tail copula $R$. We propose a procedure for constructing asymptotically distribution-free goodness-of-fit tests for the tail copula $R$. The procedure is based on a transformation of a suitable empirical process derived from a semi-parametric estimator of $R$. The transformed empirical process converges weakly to a standard Wiener process, paving the way for a multitude of asymptotically distribution-free goodnessof-fit tests. We also extend our results to the $m$-variate $(m>2)$ case. In a simulation study we show that the limit theorems provide good approximations for finite samples and that tests based on the transformed empirical process have high power.
\end{abstract}

1. Introduction. Let $\left(X_{1}, Y_{1}\right), \ldots,\left(X_{n}, Y_{n}\right)$ be an i.i.d. sample from a bivariate distribution function (d.f.) $F$ with marginal d.f.'s $F_{1}(x)=F(x, \infty)$ and $F_{2}(y)=F(\infty, y)$ for $x, y \in \mathbb{R}$. Suppose that $F$ is in the max-domain of attraction of some bivariate d.f. $G$ with nondegenerate marginals. That is, suppose that there exist normalizing sequences $a_{1}(n), a_{2}(n)>0$ and $b_{1}(n), b_{2}(n) \in$ $\mathbb{R}$ such that

$$
P\left(\frac{\bigvee_{i=1}^{n} X_{i}-b_{1}(n)}{a_{1}(n)} \leq x, \frac{\bigvee_{i=1}^{n} Y_{i}-b_{2}(n)}{a_{2}(n)} \leq y\right) \rightarrow G(x, y)
$$

\footnotetext{
Received November 2013; revised December 2014.

${ }^{1}$ Supported in part by the Netherlands Organization for Scientific Research under grant NWO Vidi 2009.

AMS 2000 subject classifications. Primary 62G10, 62G20, 62G32; secondary 62F03.

Key words and phrases. Extreme value theory, tail dependence, goodness-of-fit testing, martingale transform.
}

This is an electronic reprint of the original article published by the Institute of Mathematical Statistics in The Annals of Statistics, 2015, Vol. 43, No. 2, 878-902. This reprint differs from the original in pagination and typographic detail. 
as $n \rightarrow \infty$, for all continuity points $(x, y) \in \mathbb{R}^{2}$ of $G$. Of course, (1) is equivalent to

$$
F^{n}\left(a_{1}(n) x+b_{1}(n), a_{2}(n) y+b_{2}(n)\right) \rightarrow G(x, y),
$$

and the d.f. $G$ is, by definition, an extreme value d.f.

It is a classical result in extreme value theory [see de Haan and Ferreira (2006), Theorem 1.1.3] that the normalizing sequences $a_{1}, b_{1}$ and $a_{2}, b_{2}$ can be chosen in such a way that the marginal d.f.'s $G_{1}(x)=G(x, \infty)$ and $G_{2}(y)=G(\infty, y)$ are of the form

$$
\begin{array}{ll}
G_{1}(x)=\exp \left\{-\left(1+\gamma_{1} x\right)^{-1 / \gamma_{1}}\right\}, & 1+\gamma_{1} x>0, \\
G_{2}(y)=\exp \left\{-\left(1+\gamma_{2} y\right)^{-1 / \gamma_{2}}\right\}, & 1+\gamma_{2} y>0
\end{array}
$$

for some $\gamma_{1}, \gamma_{2} \in \mathbb{R}$. [Here, and in the rest of the paper, expressions of the form $(1+\gamma \cdot)^{1 / \gamma}$ should be interpreted as $\exp (\cdot)$ when $\gamma=0$.] We will assume throughout that the normalizing sequences are chosen in this way. Then $G$ is necessarily continuous, as it has continuous marginal d.f.'s, and the equivalent convergences (1) and (2) hold for all $(x, y) \in[-\infty, \infty]^{2}$. Also, $G$ can be fully characterized by the marginal extreme value indices $\gamma_{1}, \gamma_{2}$ and a description of the dependence structure between the marginal d.f.'s $G_{1}$ and $G_{2}$. Due to de Haan and Resnick (1977), it is known that the class of possible dependence structures for bivariate extreme value distributions does not form a finite-dimensional parametric family. Nevertheless, there are various equivalent ways of describing extreme value (or tail) dependence structures, each with its own advantages in applications. For an overview, we refer to Beirlant et al. (2004), Chapter 8 or de Haan and Ferreira (2006), Part II.

In this paper, we will focus on one possible description of the bivariate tail dependence structure, namely the tail copula. For a bivariate extreme value d.f. $G$ with marginal d.f.'s as given in (3), the tail copula $R$ is defined as

$$
R(x, y)=x+y+\log G\left(\frac{x^{-\gamma_{1}}-1}{\gamma_{1}}, \frac{y^{-\gamma_{2}}-1}{\gamma_{2}}\right), \quad(x, y) \in[0, \infty)^{2} .
$$

We say that a bivariate d.f. $F$ belonging to the domain of attraction of $G$ has associated tail copula $R$. It is clear that tail copulas are not copula functions in the usual sense (since they are not distribution functions of probability measures, e.g.), yet they fully capture the asymptotic dependence structure of the component-wise maxima, just like copulas capture the dependence structure of random vectors. Indeed, it is easily checked that $G(x, y)=C_{G}\left(G_{1}(x), G_{2}(y)\right)$, with

$$
C_{G}(u, v)=u v \exp \{R(-\log u,-\log v)\}, \quad(u, v) \in(0,1]^{2} .
$$


In other words, $G$ is the unique d.f. characterized by the marginal d.f.'s (3) and the copula (5).

We conclude that the asymptotic joint behavior of the standardized component-wise maxima $\bigvee_{i=1}^{n} X_{i}$ and $\bigvee_{i=1}^{n} Y_{i}$ is fully characterized by the marginal extreme value indices $\gamma_{1}, \gamma_{2}$ appearing in (3) and the tail copula $R$ defined in (4). Statistical inference about extreme value indices is a classical and well-studied problem in univariate extreme value theory; we refer to Beirlant et al. (2004), Chapters 4 and 5 or de Haan and Ferreira (2006), Chapter 3 for more information. There is also a growing literature on inference about the tail dependence structure; see Beirlant et al. (2004), Chapter 9 or de Haan and Ferreira (2006), Chapter 7, for an overview. In this paper, we will focus on inference about $R$. In particular, we will propose a semi-parametric estimator of $R$, describe a transformation of the empirical process derived from it and demonstrate how this transformed empirical process can serve as a basis to construct asymptotically distribution-free goodness-of-fit tests for $R$.

1.1. More on tail dependence. The tail copula $R$ can also be obtained (and its domain extended) in the following way from the d.f. $F$ :

$$
\begin{aligned}
& R(x, y)=\lim _{t \rightarrow \infty} t P\left(1-F_{1}(X)<x / t, 1-F_{2}(Y)<y / t\right), \\
&(x, y) \in[0, \infty]^{2} \backslash\{(\infty, \infty)\},
\end{aligned}
$$

where $(X, Y)$ denotes a random vector with d.f. $F$. If $F$ has continuous marginals, (6) can also be written as

$$
R(x, y)=\lim _{t \rightarrow \infty} t \bar{C}_{F}(x / t, y / t), \quad(x, y) \in[0, \infty]^{2} \backslash\{(\infty, \infty)\},
$$

where $\bar{C}_{F}$ denotes the "survival copula" of $F$, that is, the copula associated with $(-X,-Y)$. Observe that $R(x, \infty)=R(\infty, x)=x$ for all $x \in[0, \infty)$ and $0 \leq R(x, y) \leq x \wedge y$ for all $(x, y) \in[0, \infty]^{2} \backslash\{(\infty, \infty)\}$. It is also clear from (6) that $R$ is homogeneous of order 1 , so the restriction of $R$ on, for example, $[0,1]^{2}$ determines $R$ on its entire domain. The characterization (6) stems from Huang (1992), where it is used to derive a nonparametric estimator for $R$. We will use an alternative, semi-parametric estimator better suited for our purposes; see Section 2.

The value $R(1,1)$ is known in the applied extreme value literature as the (upper) tail dependence coefficient and is widely used as a measure of tail dependence. When $R(1,1)=0$, which is equivalent to $R \equiv 0$ on $[0, \infty)^{2}$, we call $X$ and $Y$ tail independent. When $R(1,1)>0$, we say that $X$ and $Y$ exhibit tail dependence. Other ways of describing the tail dependence structure include the stable tail dependence function, the exponent measure, the spectral measure and the Pickands dependence function; see the monographs Kotz 
and Nadarajah (2000), Beirlant et al. (2004), de Haan and Ferreira (2006) and the many references therein.

We also note here that the function $R$ generates a $\sigma$-finite measure, which we will also, without confusion, denote by $R$, on Borel subsets of $[0, \infty]^{2} \backslash$ $\{(\infty, \infty)\}$, through the identity

$$
R([0, x] \times[0, y]):=R(x, y), \quad(x, y) \in[0, \infty]^{2} \backslash\{(\infty, \infty)\} .
$$

1.2. Goodness-of-fit testing. In the literature and in practice, often a parametric model is used for the tail copula $R$; see, for example, Coles and Tawn (1991) or Joe, Smith and Weissman (1992). Testing the goodness-of-fit of the parametric model to a given data sample is therefore an important problem with abundant applications in many fields such as insurance and risk management, finance and econometrics and hydrology and meteorology. In this paper, we develop a procedure for constructing asymptotically distribution-free goodness-of-fit tests for the tail copula $R$ of a bivariate d.f. $F$. We consider null hypotheses of the form $R \in \mathcal{R}=\left\{R_{\boldsymbol{\theta}}: \boldsymbol{\theta} \in \Theta\right\}$, where $\mathcal{R}$ is a parametric family of tail copulas. Of course, by taking the parameter space $\Theta$ to consist of a single point, our results can also be used to test the goodness-of-fit of a fully specified tail copula to the data.

Our approach is based on a semi-parametric estimator $\widehat{R}_{n}$ of $R$, to be defined below. We consider a suitably normalized difference, $\widehat{\eta}_{n}$, between $\widehat{R}_{n}$ and $R_{\widehat{\boldsymbol{\theta}}}$ (with $\widehat{\boldsymbol{\theta}}$ denoting a suitable estimator of $\boldsymbol{\theta}$ ), and we show that, under the null hypothesis, a proper transformation of $\widehat{\eta}_{n}$ converges weakly to a standard Wiener process $W$. This fundamental result allows one to construct a myriad of goodness-of-fit tests based on comparisons of appropriate functionals of $\widehat{\eta}_{n}$ (the test statistics the practitioner may prefer to use) with the same functionals of $W$. We emphasize that, since $W$ is a standard Wiener process, our approach leads to asymptotically distribution-free goodness-of-fit tests: under the null hypothesis, the asymptotic distributions of the test statistics do not depend on $\mathcal{R}$ or the true $\boldsymbol{\theta}$. A simulation study confirms the applicability of our approach for finite samples.

Testing (and estimation) problems for the tail copula have been studied in the recent literature. In Einmahl, de Haan and Li (2006) the existence of $R$ is tested, rather than its membership of a parametric family. In de Haan, Neves and Peng (2008) a specific Cramér-von Mises type statistic for $R \in\left\{R_{\boldsymbol{\theta}}: \boldsymbol{\theta} \in \Theta\right\}$ is studied for two-dimensional data and a onedimensional parameter; the test statistic has a complicated limiting distribution under the null hypothesis. In Einmahl, Krajina and Segers (2012) it is assumed that $R \in\left\{R_{\boldsymbol{\theta}}: \boldsymbol{\theta} \in \Theta\right\}$, and it is then tested if $R$ is a member of a smaller parametric family, obtained by setting some components of $\boldsymbol{\theta}$ equal to fixed values. 
The remainder of the paper is organized as follows. In Section 2, we describe the semi-parametric estimator $\widehat{R}_{n}$, introduce the empirical process $\widehat{\eta}_{n}$, which is the normalized difference between $\widehat{R}_{n}$ and $R_{\widehat{\boldsymbol{\theta}}}$, and describe the weak limit $\hat{\eta}$ of $\widehat{\eta}_{n}$ as $n \rightarrow \infty$. In Section 3, we describe our key transformation from $\widehat{\eta}$ into a standard Wiener process. In Section 4, we show that the same transformation (or rather an empirical version of it, with unknown parameters replaced by estimators) applied to $\widehat{\eta}_{n}$ produces a process whose weak limit is a standard Wiener process. This is our main result. In Section 5 , we extend this result to the $m$-dimensional setting, for $m>2$. Finally, in Section 6, we demonstrate through Monte Carlo simulations the applicability of our limit theorems in finite samples and the high power properties of tests based on our results. Proofs are deferred to Section 7. The paper is supplemented by an online appendix, see Can et al. (2015), which contains some details suppressed in Section 2 as well as technical specifics about the Monte Carlo simulations, including the computer code.

2. An estimator for $\boldsymbol{R}$ and its asymptotic behavior. As in Section 1, we let $\left(X_{1}, Y_{1}\right), \ldots,\left(X_{n}, Y_{n}\right)$ denote an i.i.d. sample from a bivariate d.f. $F$ with marginal d.f.'s $F_{1}$ and $F_{2}$. We assume that the bivariate domain of attraction condition (1) holds, with the normalizing sequences $a_{1}, b_{1}$ and $a_{2}, b_{2}$ chosen such that the marginal d.f.'s $G_{1}$ and $G_{2}$ are as in (3). Taking logarithms in (2), and replacing the discrete index $n$ by a continuous index $t>0$, we obtain

$$
\lim _{t \rightarrow \infty} t\left[1-F\left(a_{1}(t) x+b_{1}(t), a_{2}(t) y+b_{2}(t)\right)\right]=-\log G(x, y), \quad(x, y) \in \mathbb{R}^{2} .
$$

Combining this with the corresponding marginal results and (5) leads to

$$
\begin{array}{rl}
\lim _{t \rightarrow \infty} t & P\left(X_{1}>a_{1}(t) x+b_{1}(t), Y_{1}>a_{2}(t) y+b_{2}(t)\right) \\
& =R\left(-\log G_{1}(x),-\log G_{2}(y)\right),
\end{array}
$$

or equivalently,

$$
\lim _{t \rightarrow \infty} t P\left(\widetilde{X}_{1}(t) \leq x, \widetilde{Y}_{1}(t) \leq y\right)=R(x, y)
$$

with

$$
\begin{aligned}
& \tilde{X}_{i}(t)=\left[\left(1+\gamma_{1} \frac{X_{i}-b_{1}(t)}{a_{1}(t)}\right) \vee 0\right]^{-1 / \gamma_{1}}, \\
& \tilde{Y}_{i}(t)=\left[\left(1+\gamma_{2} \frac{Y_{i}-b_{2}(t)}{a_{2}(t)}\right) \vee 0\right]^{-1 / \gamma_{2}},
\end{aligned}
$$

for $i=1, \ldots, n$. We conclude that if we let $k=k(n)$ denote an intermediate sequence, that is, $k \rightarrow \infty$ and $k / n \rightarrow 0$ as $n \rightarrow \infty$, then

$$
R_{n}(x, y):=\frac{n}{k} P\left(\tilde{X}_{i}(n / k) \leq x, \tilde{Y}_{i}(n / k) \leq y\right) \rightarrow R(x, y)
$$


as $n \rightarrow \infty$, for all $(x, y) \in[0, \infty)^{2}$.

We estimate $R_{n}$ and hence $R$ by replacing the unknown quantities $a_{j}(n / k)$, $b_{j}(n / k)$ and $\gamma_{j}, j=1,2$, by appropriate estimators $\widehat{a}_{j}(n / k), \widehat{b}_{j}(n / k)$ and $\widehat{\gamma}_{j}$, and the probability $P$ by the corresponding empirical measure. We define, therefore,

$$
\begin{aligned}
& \widehat{X}_{i}(n / k)=\left[\left(1+\widehat{\gamma}_{1} \frac{X_{i}-\widehat{b}_{1}(n / k)}{\widehat{a}_{1}(n / k)}\right) \vee 0\right]^{-1 / \widehat{\gamma}_{1}}, \\
& \widehat{Y}_{i}(n / k)=\left[\left(1+\widehat{\gamma}_{2} \frac{Y_{i}-\widehat{b}_{2}(n / k)}{\widehat{a}_{2}(n / k)}\right) \vee 0\right]^{-1 / \widehat{\gamma}_{2}}
\end{aligned}
$$

and

$$
\widehat{R}_{n}(x, y)=\frac{1}{k} \sum_{i=1}^{n} \mathbb{1}_{\left\{\widehat{X}_{i}(n / k) \leq x, \widehat{Y}_{i}(n / k) \leq y\right\}}
$$

for $(x, y) \in[0, \infty)^{2}$; cf. de Haan and Resnick (1993).

We consider the empirical process

$$
\eta_{n}(x, y)=\sqrt{k}\left[\widehat{R}_{n}(x, y)-R(x, y)\right], \quad(x, y) \in[0, \infty)^{2} .
$$

We will establish the asymptotic behavior of $\eta_{n}$ on $[\delta, T]^{2}$, for any $0<\delta<$ $T<\infty$, but we introduce some definitions and assumptions first. Note that from now on we will omit the arguments $(n / k)$ where appropriate, for ease of notation.

Let $V_{R}(x, y)$ denote a Wiener process on $[0, \infty]^{2} \backslash\{(\infty, \infty)\}$ with "time" $R$, that is, a zero-mean Gaussian process with covariance

$$
E\left[V_{R}(x, y) V_{R}\left(x^{\prime}, y^{\prime}\right)\right]=R\left(x \wedge x^{\prime}, y \wedge y^{\prime}\right) .
$$

Also write [cf. (10)]

$$
T_{n}(x, y)=\frac{1}{k} \sum_{i=1}^{n} \mathbb{1}_{\left\{\widetilde{X}_{i} \leq x, \widetilde{Y}_{i} \leq y\right\}}, \quad(x, y) \in[0, \infty)^{2} .
$$

It is known, by Einmahl, de Haan and Sinha (1997), Lemma 3.1, that $\sqrt{k}\left(T_{n}-R_{n}\right) \Rightarrow V_{R}$ in $D\left([\delta, T]^{2}\right)$, where " $\Rightarrow$ " denotes weak convergence and $D\left([\delta, T]^{2}\right)$ denotes the Skorohod space of functions defined on $[\delta, T]^{2}$.

In order to leave the estimators $\widehat{a}_{j}, \widehat{b}_{j}$ and $\widehat{\gamma}_{j}, j=1,2$, general at this stage, we simply assume that they are chosen in such a way that:

A1. For some 6 -variate random vector $\left(A_{1}, A_{2}, B_{1}, B_{2}, \Gamma_{1}, \Gamma_{2}\right)$, we have the joint weak convergence

$$
\begin{gathered}
\sqrt{k}\left(T_{n}-R_{n}, \frac{\widehat{a}_{1}}{a_{1}}-1, \frac{\widehat{a}_{2}}{a_{2}}-1, \frac{\widehat{b}_{1}-b_{1}}{a_{1}}, \frac{\widehat{b}_{2}-b_{2}}{a_{2}}, \widehat{\gamma}_{1}-\gamma_{1}, \widehat{\gamma}_{2}-\gamma_{2}\right) \\
\Rightarrow\left(V_{R}, A_{1}, A_{2}, B_{1}, B_{2}, \Gamma_{1}, \Gamma_{2}\right)
\end{gathered}
$$


in $D\left([\delta, T]^{2}\right) \times \mathbb{R}^{6}$.

Assumption A1 is fulfilled for, for example, the moment estimators of $\gamma_{j}$, $a_{j}$ and $b_{j}$, provided that $k$ is chosen appropriately; see de Haan and Ferreira (2006), Sections 4.2 and 3.5. We further assume the following:

A2. The partial derivatives

$$
R_{(1)}(x, y):=\frac{\partial R}{\partial x}, \quad R_{(2)}(x, y):=\frac{\partial R}{\partial y}
$$

exist and are continuous on $(0, \infty)^{2}$.

A3. The sequence $k$ is chosen such that

$$
\sqrt{k} \sup _{(x, y) \in[\delta / 2, T+1]^{2}}\left|R_{n}(x, y)-R(x, y)\right| \rightarrow 0 .
$$

Finally, for $j=1,2$, we define the following functions on $(0, \infty)$ :

$$
\begin{aligned}
& f_{j}(x)= \begin{cases}\frac{x\left(x^{\gamma_{j}}-1\right)}{\gamma_{j}}, & \gamma_{j} \neq 0, \\
x \log x, & \gamma_{j}=0,\end{cases} \\
& g_{j}(x)=-x^{\gamma_{j}+1}, \\
& h_{j}(x)= \begin{cases}\frac{x\left(1-x^{\gamma_{j}}\right)}{\gamma_{j}^{2}}+\frac{x \log x}{\gamma_{j}}, & \gamma_{j} \neq 0, \\
-\left(x \log ^{2} x\right) / 2, & \gamma_{j}=0 .\end{cases}
\end{aligned}
$$

We are now ready to state the basic convergence result for $\eta_{n}$.

Theorem 2.1. Let $0<\delta<T<\infty$. If assumptions A1-A3 hold, then

$$
\begin{aligned}
\eta_{n}(x, y) \Rightarrow & V_{R}(x, y)+R_{(1)}(x, y)\left[f_{1}(x) A_{1}+g_{1}(x) B_{1}+h_{1}(x) \Gamma_{1}\right] \\
& +R_{(2)}(x, y)\left[f_{2}(y) A_{2}+g_{2}(y) B_{2}+h_{2}(y) \Gamma_{2}\right] \\
= & : \eta(x, y)
\end{aligned}
$$

in $D\left([\delta, T]^{2}\right)$.

REMARK. Note that we take $\delta>0$, since the result does not hold true in general for $\delta=0$ : the functions in (16) are unbounded near zero for $\gamma_{j}<-1$. This theorem is very similar to Theorem 5.1 in de Haan and Resnick (1993), where instead of $R$ the stable tail dependence function $l(x, y)=x+y-$ $R(x, y)$ is estimated. We nevertheless offer a detailed proof of Theorem 2.1 in Can et al. (2015), since the statement and proof of Theorem 5.1 in de Haan and Resnick (1993) are not completely correct; in particular, our $\delta$ is taken to be 0 there. 
2.1. Parametric empirical process. Now suppose that the tail copula $R$ is a member of some parametric family of tail copulas, $\mathcal{R}=\left\{R_{\boldsymbol{\theta}}: \boldsymbol{\theta} \in \Theta\right\}$, where $\Theta$ is an open subset of $\mathbb{R}^{d}$. Then there is a $\boldsymbol{\theta}_{0}=\left(\theta_{01}, \ldots, \theta_{0 d}\right)^{\top} \in \Theta$ such that $R=R_{\boldsymbol{\theta}_{0}}$. Let $\widehat{\boldsymbol{\theta}}=\left(\widehat{\theta}_{1}, \ldots, \widehat{\theta}_{d}\right)^{\top}$ denote an estimator of $\boldsymbol{\theta}_{0}$, and consider the empirical process

$$
\widehat{\eta}_{n}(x, y)=\sqrt{k}\left[\widehat{R}_{n}(x, y)-R_{\widehat{\boldsymbol{\theta}}}(x, y)\right], \quad(x, y) \in[0, \infty)^{2},
$$

the parametric version of (13). Our next result will establish the asymptotic behavior of $\widehat{\eta}_{n}$. Since

$$
\widehat{\eta}_{n}(x, y)=\eta_{n}(x, y)+\sqrt{k}\left[R_{\boldsymbol{\theta}_{0}}(x, y)-R_{\widehat{\boldsymbol{\theta}}}(x, y)\right],
$$

the asymptotic behavior of $\widehat{\eta}_{n}$ is an easy consequence of Theorem 2.1, under proper assumptions. We state those assumptions below.

B1. There is a $(6+d)$-variate random vector $\left(A_{1}, A_{2}, B_{1}, B_{2}, \Gamma_{1}, \Gamma_{2}, \boldsymbol{\zeta}\right)$ such that

$$
\begin{aligned}
& \sqrt{k}\left(T_{n}-R_{n}, \frac{\widehat{a}_{1}}{a_{1}}-1, \frac{\widehat{a}_{2}}{a_{2}}-1, \frac{\widehat{b}_{1}-b_{1}}{a_{1}}, \frac{\widehat{b}_{2}-b_{2}}{a_{2}}, \widehat{\gamma}_{1}-\gamma_{1}, \widehat{\gamma}_{2}-\gamma_{2}, \boldsymbol{\theta}_{0}-\widehat{\boldsymbol{\theta}}\right) \\
& \quad \Rightarrow\left(V_{R}, A_{1}, A_{2}, B_{1}, B_{2}, \Gamma_{1}, \Gamma_{2}, \boldsymbol{\zeta}\right)
\end{aligned}
$$

in $D\left([\delta, T]^{2}\right) \times \mathbb{R}^{6+d}$.

B2. The first-order partial derivatives

$$
\begin{aligned}
R_{\boldsymbol{\theta}(1)}(x, y) & =\frac{\partial}{\partial x} R_{\boldsymbol{\theta}}(x, y), \quad R_{\boldsymbol{\theta}(2)}(x, y)=\frac{\partial}{\partial y} R_{\boldsymbol{\theta}}(x, y), \\
\dot{R}_{\boldsymbol{\theta}}(x, y) & =\left(\frac{\partial}{\partial \theta_{1}} R_{\boldsymbol{\theta}}(x, y), \ldots, \frac{\partial}{\partial \theta_{d}} R_{\boldsymbol{\theta}}(x, y)\right)^{\top}
\end{aligned}
$$

exist and are continuous for $(x, y, \boldsymbol{\theta}) \in(0, \infty)^{2} \times B\left(\boldsymbol{\theta}_{0}\right)$, for some neighborhood $B\left(\boldsymbol{\theta}_{0}\right)$ of $\boldsymbol{\theta}_{0}$ in $\Theta$.

B3. The sequence $k$ is chosen such that

$$
\sqrt{k} \sup _{(x, y) \in[\delta / 2, T+1]^{2}}\left|R_{n}(x, y)-R_{\boldsymbol{\theta}_{0}}(x, y)\right| \rightarrow 0 .
$$

Note that B3 is the same as A3; we restate it here for ease of presentation. Also note that by virtue of $\mathrm{B} 2$ the second term on the right-hand side of (19) is asymptotically equal in probability to

$$
\dot{R}_{\boldsymbol{\theta}_{0}}^{\top}(x, y) \sqrt{k}\left(\boldsymbol{\theta}_{0}-\widehat{\boldsymbol{\theta}}\right),
$$

which, by B1, converges weakly to $\dot{R}_{\boldsymbol{\theta}_{0}}^{\top}(x, y) \boldsymbol{\zeta}$. Thus we obtain the following corollary to Theorem 2.1. 
Corollary 2.2. Let $0<\delta<T<\infty$. If assumptions B1-B3 hold, then

$$
\begin{aligned}
\widehat{\eta}_{n}(x, y) \Rightarrow & V_{R_{\boldsymbol{\theta}_{0}}}(x, y)+R_{\boldsymbol{\theta}_{0}(1)}(x, y)\left[f_{1}(x) A_{1}+g_{1}(x) B_{1}+h_{1}(x) \Gamma_{1}\right] \\
& +R_{\boldsymbol{\theta}_{0}(2)}(x, y)\left[f_{2}(y) A_{2}+g_{2}(y) B_{2}+h_{2}(y) \Gamma_{2}\right] \\
& +\dot{R}_{\boldsymbol{\theta}_{0}}^{\top}(x, y) \boldsymbol{\zeta} \\
=: & \widehat{\eta}(x, y)
\end{aligned}
$$

in $D\left([\delta, T]^{2}\right)$.

3. Transforming $\widehat{\eta}$ into a standard Wiener process. The limiting process $\widehat{\eta}$ in $(22)$ is of the general form

$$
\xi(x, y)=V_{R}(x, y)+\sum_{j=1}^{\nu} Q_{j}(x, y) Z_{j},
$$

where $V_{R}$ denotes a Wiener process with time $R, \nu$ is a fixed integer, $Q_{1}, \ldots, Q_{\nu}$ are deterministic functions mapping $[\delta, T]^{2}$ into $\mathbb{R}$ and $Z_{1}, \ldots, Z_{\nu}$ are random variables.

It will be more convenient to consider the set-indexed version of (23),

$$
\xi(B)=V_{R}(B)+\sum_{j=1}^{\nu} Q_{j}(B) Z_{j}=: V_{R}(B)+\mathbf{Q}^{\top}(B) \mathbf{Z},
$$

where $B$ is a Borel subset of $[\delta, T]^{2}, V_{R}$ is a set-indexed Wiener process with time measure $R$ and $Q_{1}, \ldots, Q_{\nu}$ are deterministic signed measures. In the right-hand side of $(24), \mathbf{Q}(B)$ denotes the column vector consisting of $Q_{1}(B), \ldots, Q_{\nu}(B)$ and $\mathbf{Z}$ denotes the column vector consisting of $Z_{1}, \ldots, Z_{\nu}$.

We will state a general transformation result about set-indexed processes $\xi$ of the form (24), which we will then apply to the process $\widehat{\eta}$ in (22). The transformation is a suitable extension of the "innovation martingale transform" first discussed in Khmaladze $(1981,1988,1993)$ in connection with parametric goodness-of-fit testing for univariate and multivariate distribution functions; see, in particular, Khmaladze (1993), Theorem 3.9. A good summary of the innovation martingale transform idea can be found in Koul and Swordson (2011); for a variety of statistical applications we refer to McKeague, Nikabadze and Sun (1995), Nikabadze and Stute (1997), Stute, Thies and Zhu (1998), Koenker and Xiao (2002, 2006), Khmaladze and Koul (2004, 2009), Delgado, Hidalgo and Velasco (2005) and Dette and Hetzler (2009), among others.

As in Khmaladze (1993), we will call a collection of subsets $\left\{A_{u}: 0 \leq u \leq\right.$ $1\}$ of $[\delta, T]^{2}$ a scanning family over $[\delta, T]^{2}$ if the following hold:

(i) $\operatorname{Leb}\left(A_{0}\right)=0, \operatorname{Leb}\left(A_{1}\right)=(T-\delta)^{2}$, 
(ii) $A_{u} \subset A_{u^{\prime}}$ if $u \leq u^{\prime}$,

(iii) $\operatorname{Leb}\left(A_{u^{\prime}} \backslash A_{u}\right) \rightarrow 0$ if $u^{\prime} \downarrow u$,

with Leb denoting Lebesgue measure. Note that for any $j \in\{1, \ldots, \nu\}$ and Borel subset $B$ of $[\delta, T]^{2}$, the function $u \mapsto Q_{j}\left(B \cap A_{u}\right)$ generates a signed measure on $[0,1]$.

TheOREm 3.1. Let $\xi$ be a set-indexed process of the form (24). Suppose there are functions $q_{j}:[\delta, T]^{2} \rightarrow \mathbb{R}, 1 \leq j \leq \nu$ that are square-integrable with respect to $R$ and that satisfy

$$
Q_{j}(B)=\iint_{B} q_{j}(x, y) \mathrm{d} R(x, y), \quad 1 \leq j \leq \nu,
$$

for any Borel set $B \subset[\delta, T]^{2}$. Let $\left\{A_{u}: 0 \leq u \leq 1\right\}$ be a scanning family over $[\delta, T]^{2}$. Then the process

$$
W_{R}(B)=\xi(B)-\int_{0}^{1} \mathbf{Q}^{\top}\left(B \cap A_{\mathrm{d} u}\right) \mathbf{I}^{-1}\left(A_{u}^{c}\right) \iint_{A_{u}^{c}} \mathbf{q}(x, y) \mathrm{d} \xi(x, y)
$$

is a Wiener process with time $R$, where $\mathbf{q}(x, y)$ denotes the column vector consisting of $q_{1}(x, y), \ldots, q_{\nu}(x, y)$, and the matrices $\mathbf{I}\left(A_{u}^{c}\right)$ are defined by

$$
\mathbf{I}\left(A_{u}^{c}\right)=\iint_{A_{u}^{c}} \mathbf{q}(x, y) \mathbf{q}^{\top}(x, y) \mathrm{d} R(x, y), \quad u \in[0,1)
$$

and are assumed to be invertible.

Now let us return to the setup of Section 2.1. We state the following assumption.

B4. For each $\boldsymbol{\theta} \in \Theta$, the measure $R_{\boldsymbol{\theta}}$ can be decomposed as $R_{\boldsymbol{\theta}}=R_{\boldsymbol{\theta}}^{(c)}+$ $R_{\boldsymbol{\theta}}^{(s)}$, where $R_{\boldsymbol{\theta}}^{(s)}$ satisfies $R_{\boldsymbol{\theta}}^{(s)}\left([0, \infty)^{2}\right)=0$ and $R_{\boldsymbol{\theta}}^{(c)}$ is absolutely continuous with respect to the Lebesgue measure on $(0, \infty)^{2}$, with a positive density $r_{\boldsymbol{\theta}}$ that has continuous first-order partial derivatives with respect to $x, y, \theta_{1}, \ldots, \theta_{d}$ for all $(x, y, \boldsymbol{\theta}) \in(0, \infty)^{2} \times B\left(\boldsymbol{\theta}_{0}\right)$, for some neighborhood $B\left(\boldsymbol{\theta}_{0}\right)$ of $\boldsymbol{\theta}_{0}$ in $\Theta$.

Note that B4 allows arbitrarily large masses on the "axes at infinity" $\{(x, \infty): x \geq 0\} \cup\{(\infty, y): y \geq 0\}$ for $R_{\boldsymbol{\theta}} \in \mathcal{R}$, but excludes the case $R_{\boldsymbol{\theta}} \equiv$ $R_{\boldsymbol{\theta}}^{(s)}$, which corresponds to (strict) tail independence.

Let us define the following functions on $[\delta, T]^{2}$, with $f_{j}, g_{j}$ and $h_{j}$ as defined in $(16)$ :

$$
\begin{array}{lll}
Q_{1}(x, y)=R_{\boldsymbol{\theta}_{0}(1)}(x, y) f_{1}(x), & & Q_{4}(x, y)=R_{\boldsymbol{\theta}_{0}(2)}(x, y) f_{2}(y), \\
Q_{2}(x, y)=R_{\boldsymbol{\theta}_{0}(1)}(x, y) g_{1}(x), & & Q_{5}(x, y)=R_{\boldsymbol{\theta}_{0}(2)}(x, y) g_{2}(y), \\
Q_{3}(x, y)=R_{\boldsymbol{\theta}_{0}(1)}(x, y) h_{1}(x), & & Q_{6}(x, y)=R_{\boldsymbol{\theta}_{0}(2)}(x, y) h_{2}(y)
\end{array}
$$


and

$$
Q_{6+i}(x, y)=\left.\frac{\partial}{\partial \theta_{i}} R_{\boldsymbol{\theta}}(x, y)\right|_{\boldsymbol{\theta}=\boldsymbol{\theta}_{0}}, \quad i=1, \ldots, d .
$$

Furthermore, let $q_{i}$ denote the Radon-Nikodym derivatives $\mathrm{d} Q_{i} / \mathrm{d} R_{\boldsymbol{\theta}_{0}}$ for $i=1, \ldots, 6+d$, or more explicitly:

$$
\begin{aligned}
& q_{1}(x, y)=f_{1}^{\prime}(x)+f_{1}(x) \frac{\partial}{\partial x} \log r_{\boldsymbol{\theta}_{0}}(x, y) \\
& q_{2}(x, y)=g_{1}^{\prime}(x)+g_{1}(x) \frac{\partial}{\partial x} \log r_{\boldsymbol{\theta}_{0}}(x, y), \\
& q_{3}(x, y)=h_{1}^{\prime}(x)+h_{1}(x) \frac{\partial}{\partial x} \log r_{\boldsymbol{\theta}_{0}}(x, y), \\
& q_{4}(x, y)=f_{2}^{\prime}(y)+f_{2}(y) \frac{\partial}{\partial y} \log r_{\boldsymbol{\theta}_{0}}(x, y), \\
& q_{5}(x, y)=g_{2}^{\prime}(y)+g_{2}(y) \frac{\partial}{\partial y} \log r_{\boldsymbol{\theta}_{0}}(x, y) \\
& q_{6}(x, y)=h_{2}^{\prime}(y)+h_{2}(y) \frac{\partial}{\partial y} \log r_{\boldsymbol{\theta}_{0}}(x, y)
\end{aligned}
$$

and

$$
q_{6+i}(x, y)=\left.\frac{\partial}{\partial \theta_{i}} \log r_{\boldsymbol{\theta}}(x, y)\right|_{\boldsymbol{\theta}=\boldsymbol{\theta}_{0}}, \quad i=1, \ldots, d .
$$

As before, $\mathbf{q}(x, y)$ will denote the column vector consisting of $q_{1}(x, y), \ldots$, $q_{6+d}(x, y)$ for $(x, y) \in[\delta, T]^{2}$.

We are now ready to apply Theorem 3.1 to $\widehat{\eta}$ in (22). Instead of arbitrary Borel sets $B$, we consider rectangles $[\delta, x] \times[\delta, y] \subset[\delta, T]^{2}$, with

$$
\widehat{\eta}([\delta, x] \times[\delta, y]):=\widehat{\eta}(x, y)-\widehat{\eta}(\delta, y)-\widehat{\eta}(x, \delta)+\widehat{\eta}(\delta, \delta) .
$$

We also introduce the scanning family $A_{u}=[\delta, T] \times[\delta,(1-u) \delta+u T]$ for $0 \leq u \leq 1$ and define the corresponding matrices

$$
\mathbf{I}(t)=\int_{\delta}^{T} \int_{t}^{T} \mathbf{q}\left(s^{\prime}, t^{\prime}\right) \mathbf{q}^{\top}\left(s^{\prime}, t^{\prime}\right) \mathrm{d} R_{\boldsymbol{\theta}_{0}}\left(s^{\prime}, t^{\prime}\right), \quad t \in[\delta, T) .
$$

REMARK. From a likelihood theory point of view, the functions $q_{1}, \ldots$, $q_{6+d}$ can be seen as score functions corresponding to the estimated values $a_{1}, a_{2}, b_{1}, b_{2}, \gamma_{1}, \gamma_{2}, \theta_{01}, \ldots, \theta_{0 d}$, and the matrix $\mathbf{I}(t)$ can be seen as a partial Fisher information matrix constructed from these score functions. 
Corollary 3.2. If assumptions $\mathrm{B} 2$ and $\mathrm{B} 4$, restricted to $\boldsymbol{\theta}=\boldsymbol{\theta}_{0}$, hold, and the matrices $\mathbf{I}(t)$ in (26) are invertible, then the process

$$
\begin{aligned}
& W_{R}([\delta, x] \times[\delta, y]) \\
& =\widehat{\eta}([\delta, x] \times[\delta, y]) \\
& \quad-\int_{\delta}^{x} \int_{\delta}^{y} \mathbf{q}^{\top}(s, t)\left(\mathbf{I}^{-1}(t) \int_{\delta}^{T} \int_{t}^{T} \mathbf{q}\left(s^{\prime}, t^{\prime}\right) \mathrm{d} \widehat{\eta}\left(s^{\prime}, t^{\prime}\right)\right) \mathrm{d} R_{\boldsymbol{\theta}_{0}}(s, t)
\end{aligned}
$$

is a Wiener process with time $R_{\boldsymbol{\theta}_{0}}$ on $[\delta, T] \times[\delta, T)$.

In order to obtain a standard Wiener process from $\widehat{\eta}$, we normalize $W_{R}$ in the usual way, as follows.

Corollary 3.3. If assumptions $\mathrm{B} 2$ and $\mathrm{B} 4$, restricted to $\boldsymbol{\theta}=\boldsymbol{\theta}_{0}$, hold, and the matrices $\mathbf{I}(t)$ in (26) are invertible, then the process

$W([\delta, x] \times[\delta, y])$

$$
\begin{aligned}
= & \int_{\delta}^{x} \int_{\delta}^{y} \frac{1}{\sqrt{r_{\boldsymbol{\theta}_{0}}(s, t)}} \mathrm{d} W_{R}([\delta, s] \times[\delta, t]) \\
= & \int_{\delta}^{x} \int_{\delta}^{y} \frac{1}{\sqrt{r_{\boldsymbol{\theta}_{0}}(s, t)}} \mathrm{d} \widehat{\eta}(s, t) \\
& -\int_{\delta}^{x} \int_{\delta}^{y} \mathbf{q}^{\top}(s, t)\left(\mathbf{I}^{-1}(t) \int_{\delta}^{T} \int_{t}^{T} \mathbf{q}\left(s^{\prime}, t^{\prime}\right) \mathrm{d} \widehat{\eta}\left(s^{\prime}, t^{\prime}\right)\right) \sqrt{r_{\boldsymbol{\theta}_{0}}(s, t)} \mathrm{d} t \mathrm{~d} s
\end{aligned}
$$

is a standard Wiener process on $[\delta, T] \times[\delta, T)$.

4. Goodness-of-fit testing. In Section 2 we introduced the parametric empirical process $\widehat{\eta}_{n}$ as the normalized difference between $R_{\widehat{\boldsymbol{\theta}}}$ and the semiparametric estimator $\widehat{R}_{n}$, and derived its weak limit $\widehat{\eta}$. In Section 3 we described a transformation from $\widehat{\eta}$ into a standard Wiener process $W$. In this section, we will apply the empirical version of the same transformation to $\widehat{\eta}_{n}$, and prove that the resulting empirical process converges weakly to a standard Wiener process. This is the main result of this paper.

Define the empirical version of $W$ in $(27)$ as follows, for $(x, y) \in[\delta, T] \times$ $[\delta, T):$

$$
\begin{aligned}
W_{n}([\delta, x] \times[\delta, y]) \\
(28)=\int_{\delta}^{x} \int_{\delta}^{y} \frac{1}{\sqrt{r_{\widehat{\boldsymbol{\theta}}}(s, t)}} \mathrm{d} \widehat{\eta}_{n}(s, t) \\
\quad-\int_{\delta}^{x} \int_{\delta}^{y} \widehat{\mathbf{q}}^{\top}(s, t)\left(\widehat{\mathbf{I}}^{-1}(t) \int_{\delta}^{T} \int_{t}^{T} \widehat{\mathbf{q}}\left(s^{\prime}, t^{\prime}\right) \mathrm{d} \widehat{\eta}_{n}\left(s^{\prime}, t^{\prime}\right)\right) \sqrt{r_{\widehat{\boldsymbol{\theta}}}(s, t)} \mathrm{d} t \mathrm{~d} s .
\end{aligned}
$$


Here, the vectors $\widehat{\mathbf{q}}$ and the matrices $\widehat{\mathbf{I}}$ are obtained by replacing the unknown marginal tail indices $\gamma_{1}, \gamma_{2}$ and the unknown parameter $\boldsymbol{\theta}_{0}$ in the definition of $\mathbf{q}$ by their estimators $\widehat{\gamma}_{1}, \widehat{\gamma}_{2}, \widehat{\boldsymbol{\theta}}$.

For functions $\varphi:[\delta, T]^{2} \rightarrow \mathbb{R}$, we introduce the seminorm

$$
\begin{aligned}
\|\varphi\|_{\mathrm{HK}}:= & V^{(2)}(\varphi)+V^{(1)}(\varphi(\cdot, \delta))+V^{(1)}(\varphi(\delta, \cdot)) \\
& +V^{(1)}(\varphi(\cdot, T))+V^{(1)}(\varphi(T, \cdot)),
\end{aligned}
$$

where $V^{(1)}$ denotes the univariate total variation over $[\delta, T]$, and $V^{(2)}$ denotes the bivariate (Vitali) total variation over $[\delta, T]^{2}$, as defined in Owen (2005), for example. The seminorm $\|\cdot\|_{\mathrm{HK}}$ is sometimes called the Hardy-Krause variation in the literature, in recognition of Hardy (1905) and Krause (1903).

For notational convenience, let us also denote

$$
\begin{aligned}
\rho_{1}(x, y, \boldsymbol{\theta}) & =\frac{\partial}{\partial x} \log r_{\boldsymbol{\theta}}(x, y), & \rho_{2}(x, y, \boldsymbol{\theta})=\frac{\partial}{\partial y} \log r_{\boldsymbol{\theta}}(x, y), \\
\rho_{2+i}(x, y, \boldsymbol{\theta}) & =\frac{\partial}{\partial \theta_{i}} \log r_{\boldsymbol{\theta}}(x, y), & i=1, \ldots, d
\end{aligned}
$$

and

$$
\Delta \rho_{j}(x, y)=\rho_{j}(x, y, \widehat{\boldsymbol{\theta}})-\rho_{j}\left(x, y, \boldsymbol{\theta}_{0}\right), \quad j=1, \ldots, 2+d .
$$

Similarly, let

$$
\sigma(x, y, \boldsymbol{\theta})=r_{\boldsymbol{\theta}}(x, y)^{-1 / 2}, \quad \Delta \sigma(x, y)=\sigma(x, y, \widehat{\boldsymbol{\theta}})-\sigma\left(x, y, \boldsymbol{\theta}_{0}\right) .
$$

We introduce the following assumption:

B5. For $j=1, \ldots, 2+d,\left\|\rho_{j}\left(x, y, \boldsymbol{\theta}_{0}\right)\right\|_{\mathrm{HK}}<\infty$ and $\left\|\Delta \rho_{j}(x, y)\right\|_{\mathrm{HK}}=o_{P}(1)$. Furthermore, $\left\|\sigma\left(x, y, \boldsymbol{\theta}_{0}\right)\right\|_{\mathrm{HK}}<\infty$ and $\|\Delta \sigma(x, y)\|_{\mathrm{HK}}=o_{P}(1)$.

Given the consistency of $\widehat{\boldsymbol{\theta}}$, which is implied by B1, a sufficient (but not necessary) condition for B5 is the existence and continuity of the partial derivatives

$$
\frac{\partial \varphi(x, \delta, \boldsymbol{\theta})}{\partial x}, \frac{\partial \varphi(x, T, \boldsymbol{\theta})}{\partial x}, \frac{\partial \varphi(\delta, y, \boldsymbol{\theta})}{\partial y}, \frac{\partial \varphi(T, y, \boldsymbol{\theta})}{\partial y}, \frac{\partial^{2} \varphi(x, y, \boldsymbol{\theta})}{\partial x \partial y}
$$

on $(x, y, \boldsymbol{\theta}) \in[\delta, T]^{2} \times B\left(\boldsymbol{\theta}_{0}\right)$, for some neighborhood $B\left(\boldsymbol{\theta}_{0}\right)$ of $\boldsymbol{\theta}_{0}$ in $\Theta$, for $\varphi=\sigma$ and $\varphi=\rho_{j}, j=1, \ldots, 2+d$.

We can now present the main result of this paper.

TheOrem 4.1. Let $0<\delta<\tau<T$, and let $W$ and $W_{n}$ be defined as in (27) and (28). If assumptions B1-B5 hold, then

$$
W_{n}([\delta, x] \times[\delta, y]) \Rightarrow W([\delta, x] \times[\delta, y])
$$

in $D\left([\delta, \tau]^{2}\right)$. 
Note that Theorem 4.1 yields that under the null hypothesis $R \in \mathcal{R}$, we obtain a distribution-free limiting process $W$ (a standard bivariate Wiener process). Hence $W_{n}$ can be used as a "test process" for producing a myriad of asymptotically distribution-free test statistics to test this null hypothesis. We will consider examples of such tests in Section 6 .

REMARK. By taking $\mathcal{R}=\left\{R_{0}\right\}$, where $R_{0}$ is a fully specified tail copula, we can use Theorem 4.1 for testing the null hypothesis $R=R_{0}$. In this case, the process $\widehat{\eta}_{n}$ in the definition of $W_{n}$ [see (28)] reduces to $\eta_{n}$ as defined in (13), $r_{\widehat{\theta}}$ reduces to $r_{0}=\mathrm{d} R_{0} / \mathrm{d}$ Leb and $\widehat{\mathbf{q}}$ and $\widehat{\mathbf{I}}$ are determined by $r_{0}$ and $\widehat{\gamma}_{1}, \widehat{\gamma}_{2}$. We will consider an example of testing $R=R_{0}$ in Section 6 .

5. Multivariate extension. In this section we extend Theorem 4.1 from the bivariate to the $m$-dimensional setting, for $m>2$. The proof will be omitted, but it follows very similar lines as in the bivariate case. In particular, Theorem 3.1 immediately generalizes to dimension $m$ and then serves as a basis for the main result of this section.

So suppose that we have an i.i.d. sample $\mathbf{X}_{1}, \ldots, \mathbf{X}_{n}$ from an $m$-variate d.f. $F$ with marginal d.f.'s $F_{1}, \ldots, F_{m}$. We write, for each $i \in\{1, \ldots, n\}$, $\mathbf{X}_{i}=\left(X_{i 1}, \ldots, X_{i m}\right)^{\top}$, where $X_{i j}$ has d.f. $F_{j}$. We assume that $F$ is in the max-domain of attraction of an $m$-variate extreme value d.f. $G$, so there exist normalizing sequences $a_{1}(n), \ldots, a_{m}(n)>0$ and $b_{1}(n), \ldots, b_{m}(n) \in \mathbb{R}$ such that

$$
P\left(\frac{\bigvee_{i=1}^{n} X_{i 1}-b_{1}(n)}{a_{1}(n)} \leq x_{1}, \ldots, \frac{\bigvee_{i=1}^{n} X_{i m}-b_{m}(n)}{a_{m}(n)} \leq x_{m}\right) \stackrel{d}{\rightarrow} G(\mathbf{x}),
$$

with $\mathbf{x}=\left(x_{1}, \ldots, x_{m}\right)^{\top} \in \mathbb{R}^{m}$. We assume, as in the bivariate case, that the sequences $a_{j}$ and $b_{j}, j=1, \ldots, m$, are chosen in such a way that $G$ has marginal d.f.'s of the form

$$
G_{j}(x)=\exp \left\{-\left(1+\gamma_{j} x\right)^{-1 / \gamma_{j}}\right\}, \quad 1+\gamma_{j} x>0,
$$

for some $\gamma_{1}, \ldots, \gamma_{m} \in \mathbb{R}$. We will denote $\gamma=\left(\gamma_{1}, \ldots, \gamma_{m}\right)^{\top}$. The d.f. $G$ is then characterized by the marginal tail indices $\gamma$ and the $m$-variate tail copula

$$
R(\mathbf{x}):=\lim _{t \rightarrow \infty} t P\left(\bigcap_{j=1}^{m}\left\{1-F_{j}\left(X_{1 j}\right) \leq x_{j} / t\right\}\right), \quad \mathbf{x} \in[0, \infty]^{m} \backslash\{\infty\},
$$

where $\infty$ denotes the point $(\infty, \ldots, \infty)$.

REMARK. In the remainder of this section we consider $R$ defined on the restricted domain $[0, \infty)^{m}$ [cf. (4)] because our processes and transformations are not defined outside this region. The bivariate tail copula $R$ defined on $[0, \infty)^{2}$ determines $R$ on the full domain $[0, \infty]^{2} \backslash\{(\infty, \infty)\}$. In contrast, for $m>2$ the tail copula $R$ defined on $[0, \infty)^{m}$ in general does not determine $R$ on the full domain $[0, \infty]^{m} \backslash\{\infty\}$. 
Let $\mathcal{R}=\left\{R_{\boldsymbol{\theta}}: \boldsymbol{\theta} \in \Theta\right\}$ denote a parametric family of $m$-variate tail copulas on $[0, \infty)^{m}$, parametrized by $\boldsymbol{\theta}=\left(\theta_{1}, \ldots, \theta_{d}\right)^{\top} \in \Theta$, an open subset of $\mathbb{R}^{d}$. Our aim is to enable the construction of tests for the null hypothesis $R \in \mathcal{R}$ against the alternative $R \notin \mathcal{R}$.

For fixed $\boldsymbol{\theta} \in \Theta, R_{\boldsymbol{\theta}}$ can be seen as an equivalence class of tail dependence structures (i.e., tail copulas defined on the full domain) containing one or more elements. Under the additional assumption that $R_{\boldsymbol{\theta}}$ puts no mass on $[0, \infty]^{m} \backslash\left(\{\infty\} \cup[0, \infty)^{m}\right), R_{\boldsymbol{\theta}}$ contains exactly one element (as in the bivariate case).

Suppose the null hypothesis holds true, with $R=R_{\boldsymbol{\theta}_{0}}$, for some $\boldsymbol{\theta}_{0} \in \Theta$. Let $\widehat{\boldsymbol{\theta}}$ denote an estimator for $\boldsymbol{\theta}_{0}$. As in Section 2, we let $k=k(n)$ denote an intermediate sequence and define the parametric empirical process

$$
\widehat{\eta}_{n}(\mathbf{x})=\sqrt{k}\left[\widehat{R}_{n}(\mathbf{x})-R_{\widehat{\boldsymbol{\theta}}}(\mathbf{x})\right], \quad \mathbf{x} \in[0, \infty)^{m},
$$

where

$$
\widehat{R}_{n}(\mathbf{x})=\frac{1}{k} \sum_{i=1}^{n} \mathbb{1}_{\bigcap_{j=1}^{m}\left\{\widehat{X}_{i j}(n / k) \leq x_{j}\right\}}, \quad \mathbf{x} \in[0, \infty)^{m},
$$

with $\widehat{X}_{i j}(n / k),(i, j) \in\{1, \ldots, n\} \times\{1, \ldots, m\}$, defined similarly as in (11). Let $R_{n}$ and $T_{n}$ denote the obvious $m$-variate extensions of (10) and (14), let $0<\delta<T<\infty$ and let $\mathrm{C} 1-\mathrm{C} 4$ denote the natural $m$-variate extensions of assumptions B1-B4 of Sections 2 and 3.

To state the analog of assumption B5 for the $m$-variate case, we extend the seminorm (29) to $m$-variate functions by induction, as follows: For any function $\varphi:[\delta, T]^{m} \rightarrow \mathbb{R}$, and $i \in\{1, \ldots, m\}$, we define $\varphi_{\delta, i}:[\delta, T]^{m-1} \rightarrow \mathbb{R}$ to be the restriction of $\varphi$ to the subset of $[\delta, T]^{m}$ with the $i$ th coordinate fixed at $\delta$, and we define $\varphi_{T, i}$ analogously. Then we let

$$
\|\varphi\|_{\mathrm{HK}}^{(m)}:=V^{(m)}(\varphi)+\sum_{i=1}^{m}\left\|\varphi_{\delta, i}\right\|_{\mathrm{HK}}^{(m-1)}+\sum_{i=1}^{m}\left\|\varphi_{T, i}\right\|_{\mathrm{HK}}^{(m-1)},
$$

with $V^{(m)}$ denoting the $m$-variate (Vitali) total variation over $[\delta, T]^{m}$ and $\|\varphi\|_{\mathrm{HK}}^{(2)}$ as defined in (29). We also let $\rho_{j}, \Delta \rho_{j}, \sigma, \Delta \sigma$ be defined as in Section 4 , for $j=1, \ldots, m+d$.

C5. For $j=1, \ldots, m+d,\left\|\rho_{j}\left(\mathbf{x}, \boldsymbol{\theta}_{0}\right)\right\|_{\mathrm{HK}}^{(m)}<\infty$ and $\left\|\Delta \rho_{j}(\mathbf{x})\right\|_{\mathrm{HK}}^{(m)}=o_{P}(1)$. Furthermore, $\left\|\sigma\left(\mathbf{x}, \boldsymbol{\theta}_{0}\right)\right\|_{\mathrm{HK}}^{(m)}<\infty$ and $\|\Delta \sigma(\mathbf{x})\|_{\mathrm{HK}}^{(m)}=o_{P}(1)$.

Now, let us introduce the functions $Q_{j}$ and $q_{j}=\mathrm{d} Q_{j} / \mathrm{d} R_{\boldsymbol{\theta}_{0}}$, for $j=$ $1, \ldots, 3 m+d$, as the natural $m$-variate extensions of the bivariate functions introduced before Corollary 3.2, and let us denote by $\mathbf{q}(\mathbf{x})$ the column vector consisting of $q_{1}(\mathbf{x}), \ldots, q_{3 m+d}(\mathbf{x})$. Further, let us write $[\boldsymbol{\delta}, \mathbf{x}]=$ 


$$
\begin{gathered}
{\left[\delta, x_{1}\right] \times \cdots \times\left[\delta, x_{m}\right], S_{t}=[\delta, T]^{m-1} \times(t, T], \text { and introduce matrices }} \\
\mathbf{I}(t)=\int_{S_{t}} \mathbf{q}(\mathbf{s}) \mathbf{q}^{\top}(\mathbf{s}) \mathrm{d} R_{\boldsymbol{\theta}_{0}}(\mathbf{s}), \quad t \in[\delta, T),
\end{gathered}
$$

which are assumed to be invertible. Then the $m$-variate analog of the transformed empirical process $W_{n}$ in (28) is

$$
\begin{aligned}
W_{n}([\boldsymbol{\delta}, \mathbf{x}])= & \int_{[\boldsymbol{\delta}, \mathbf{x}]} \frac{1}{\sqrt{r_{\widehat{\boldsymbol{\theta}}}(\mathbf{s})}} \mathrm{d} \widehat{\eta}_{n}(\mathbf{s}) \\
& -\int_{[\boldsymbol{\delta}, \mathbf{x}]} \widehat{\mathbf{q}}^{\top}(\mathbf{s})\left(\widehat{\mathbf{I}}^{-1}(t) \int_{S_{t}} \widehat{\mathbf{q}}\left(\mathbf{s}^{\prime}\right) \mathrm{d} \widehat{\eta}_{n}\left(\mathbf{s}^{\prime}\right)\right) \sqrt{r_{\widehat{\boldsymbol{\theta}}}(\mathbf{s})} \mathrm{d} \mathbf{s},
\end{aligned}
$$

where $\widehat{\mathbf{q}}$ and $\widehat{\mathbf{I}}$ are obtained by replacing $\boldsymbol{\gamma}$ and $\boldsymbol{\theta}_{0}$ by $\widehat{\boldsymbol{\gamma}}$ and $\widehat{\boldsymbol{\theta}}$ in the definition of $\mathbf{q}$.

We are now ready to state the multivariate analog of Theorem 4.1. As in the bivariate case, this result can be used as a basis for producing a multitude of asymptotically distribution-free goodness-of-fit tests for a parametric model $\mathcal{R}$ (as well as for a fully specified tail copula $R_{0}$ ).

Theorem 5.1. Let $m>2$. Furthermore, let $0<\delta<\tau<T$, and let $W_{n}$ be defined as in (31). If assumptions C1-C5 hold, then

$$
W_{n}([\boldsymbol{\delta}, \mathbf{x}]) \Rightarrow W([\boldsymbol{\delta}, \mathbf{x}])
$$

in $D\left([\delta, \tau]^{m}\right)$, where $W$ is a standard $m$-variate Wiener process.

6. Simulation study. In this section we consider some specific functionals of $W_{n}$ under the null and alternative hypotheses, for three bivariate models $\mathcal{R}$. We will see in Monte Carlo simulations that under the null hypothesis our limit theorems yield good approximations for finite sample size $n$, and we also find that the resulting tests have good power properties. This shows the applicability of our method.

The three models we consider are the following:

Model 1. $R(x, y)=x+y-\sqrt{x^{2}+y^{2}}$;

Model 2. $R \in \mathcal{R}=\left\{R_{\theta}: R_{\theta}(x, y)=x+y-\left(x^{1 / \theta}+y^{1 / \theta}\right)^{\theta}, \theta \in(0,1)\right\} ;$

Model 3. $R \in \mathcal{R}=\left\{R_{\psi}: R_{\psi}(x, y)=\psi\left(x+y-\sqrt{x^{2}+y^{2}}\right), \psi \in(0,1)\right\}$.

Model 2 is the widely used logistic family of tail copulas. Model 1 is a fully specified tail copula and a special case of Model 2. Model 3 is a mixture between Model 1 and the tail independence model $(R \equiv 0)$. Note that the tail copulas of Model 3 assign mass to the axes at infinity; indeed, the parameter $\psi$ determines how much mass is assigned there. 
For each model, we first generate 300 samples of size $n=1500$ from a "null hypothesis d.f." $F_{0}$ for which the model is correct. We use these samples to assess the finite-sample performance of our main convergence result, Theorem 4.1. Next, we generate, for each model, 100 samples of size $n=1500$ from an "alternative hypothesis d.f." $F_{a}$ for which the model is incorrect. These samples are used for power calculations.

In Section 6.1 below, we present the data generating distributions used for each model. Then in Section 6.2 we describe our simulation results. Additional details about the simulations, including the verification of assumptions and the computer code that was used, can be found in Can et al. (2015).

6.1. Data generating distributions. To test for Models 1 and 2 under the null hypothesis, we generate samples from the bivariate Cauchy distribution on the positive quadrant with density

$$
f_{0}(x, y)=\frac{2}{\pi\left(1+x^{2}+y^{2}\right)^{3 / 2}}, \quad(x, y) \in[0, \infty)^{2} .
$$

This distribution satisfies Model 1 , and therefore also Model 2, with $\theta=1 / 2$.

To test for Model 3 under the null hypothesis, we sample from the bivariate mixture random vector

$$
\left(I X_{1}+(1-I) X_{2}, I Y_{1}+(1-I) Y_{2}\right),
$$

where $I,\left(X_{1}, Y_{1}\right),\left(X_{2}, Y_{2}\right)$ are independent, $I \sim \operatorname{Bernoulli}(0.75),\left(X_{1}, Y_{1}\right)$ has the bivariate Cauchy distribution (32) on the positive quadrant and $\left(X_{2}, Y_{2}\right)$ is a pair of standard Cauchy absolute values coupled by the countermonotonic copula. Since $\left(X_{1}, Y_{1}\right)$ has the Model 1 tail copula and $\left(X_{2}, Y_{2}\right)$ has tail independence, mixture (33) has the Model 3 tail copula with $\psi=0.75$.

To test for Model 1 under the alternative hypothesis, we sample from a mixture random vector as in (33), where $I,\left(X_{1}, Y_{1}\right),\left(X_{2}, Y_{2}\right)$ are independent and $I \sim \operatorname{Bernoulli}(0.75)$ as before, but $\left(X_{1}, Y_{1}\right)$ has a bivariate logistic d.f. with Fréchet marginals,

$$
F(x, y)=\exp \left\{-\left[(1+x)^{-4}+(1+y)^{-4}\right]^{1 / 4}\right\}, \quad(x, y) \in(-1, \infty)^{2},
$$

and $\left(X_{2}, Y_{2}\right)$ has identical marginal d.f.'s as in (34), coupled by the countermonotonic copula. The resulting d.f. has the tail copula

$$
R(x, y)=0.75\left[x+y-\left(x^{4}+y^{4}\right)^{1 / 4}\right], \quad(x, y) \in[0, \infty)^{2} .
$$

To test for Model 2 under the alternative hypothesis, we sample from the bivariate vector

$$
\left(\lambda Z_{1}+(1-\lambda) Z_{2}, \mu Z_{1}+(1-\mu) Z_{2}\right),
$$

where $Z_{1}$ and $Z_{2}$ denote independent standard Pareto random variables, and $\lambda, \mu \in(0,1)$ are deterministic coefficients. We set $\lambda=0.95, \mu=0.65$ for 
the simulations. The random vector (35) is a simple example of the linear factor model, with associated tail copula

$$
R(x, y)=\min \{\lambda x, \mu y\}+\min \{(1-\lambda) x,(1-\mu) y\}, \quad(x, y) \in[0, \infty)^{2} .
$$

Finally, to test for Model 3 under the alternative hypothesis, we sample from the following asymmetric logistic d.f. with Fréchet marginals:

$$
\begin{aligned}
& F_{a}(x, y)=\exp \left\{-\left[\frac{1-\phi}{1+y}+\sqrt{\left.\frac{1}{(1+x)^{2}}+\frac{\phi^{2}}{(1+y)^{2}}\right]}\right.\right. \\
&(x, y) \in(-1, \infty)^{2}
\end{aligned}
$$

with $\phi=0.25$. This d.f. has the tail copula

$$
R(x, y)=x+\phi y-\sqrt{x^{2}+(\phi y)^{2}}, \quad(x, y) \in[0, \infty)^{2} .
$$

6.2. Simulation results. From each generated sample, the empirical process $W_{n}([\delta, x] \times[\delta, y])$ of $(28)$ is computed on a $200 \times 200$ grid $\mathcal{G}$ of uniform mesh length spanned over $[\delta, \tau]^{2}$, with $\delta=0.001$ and $\tau=1.001$. We take $k=250$ and $T=2$ for all computations. The estimators $\widehat{\gamma}_{j}$ and $\widehat{a}_{j}, j=1,2$, are taken to be the moment estimators [see, e.g., de Haan and Ferreira (2006), Sections 4.2 and 3.5], and we set as usual $\widehat{b}_{1}=X_{n-k: n}, \widehat{b}_{2}=Y_{n-k: n}$, with $X_{i: n}, Y_{i: n}$ denoting the marginal order statistics. To estimate the parameters $\theta$ and $\psi$ of Models 2 and 3, we use the method of moments estimator described in Einmahl, Krajina and Segers (2008), with auxiliary function $g \equiv 1$.

To compare the process $W_{n}$ to a standard Wiener process, three test statistics are computed from each path of $W_{n}$. These are:

$$
\begin{aligned}
\kappa_{n} & =\max _{(x, y) \in \mathcal{G}}\left|W_{n}([\delta, x] \times[\delta, y])\right| \quad(\text { Kolmogorov-Smirnov type) }, \\
\omega_{n}^{2} & =\|\mathcal{G}\|^{2} \sum_{(x, y) \in \mathcal{G}} W_{n}([\delta, x] \times[\delta, y])^{2} \quad \text { (Cramér-von Mises type) }, \\
A_{n}^{2} & =\|\mathcal{G}\|^{2} \sum_{(x, y) \in \mathcal{G}} \frac{W_{n}([\delta, x] \times[\delta, y])^{2}}{(x-\delta)(y-\delta)} \quad \text { (Anderson-Darling type) },
\end{aligned}
$$

where $\|\mathcal{G}\|$ denotes the mesh length of the grid $\mathcal{G}$, that is, $1 / 200$. To create benchmark distribution tables for these statistics, we also simulate 10,000 true standard Wiener process paths on the grid $\mathcal{G}$, and we compute the same test statistics for each path. We denote these statistics, computed from the true standard Wiener process, by $\kappa, \omega^{2}$ and $A^{2}$. In view of the asymptotically distribution-free nature of our approach, these benchmark tables need to be produced only once. 
DISTRIBUTION-FREE GOF TESTING FOR TAIL COPULAS
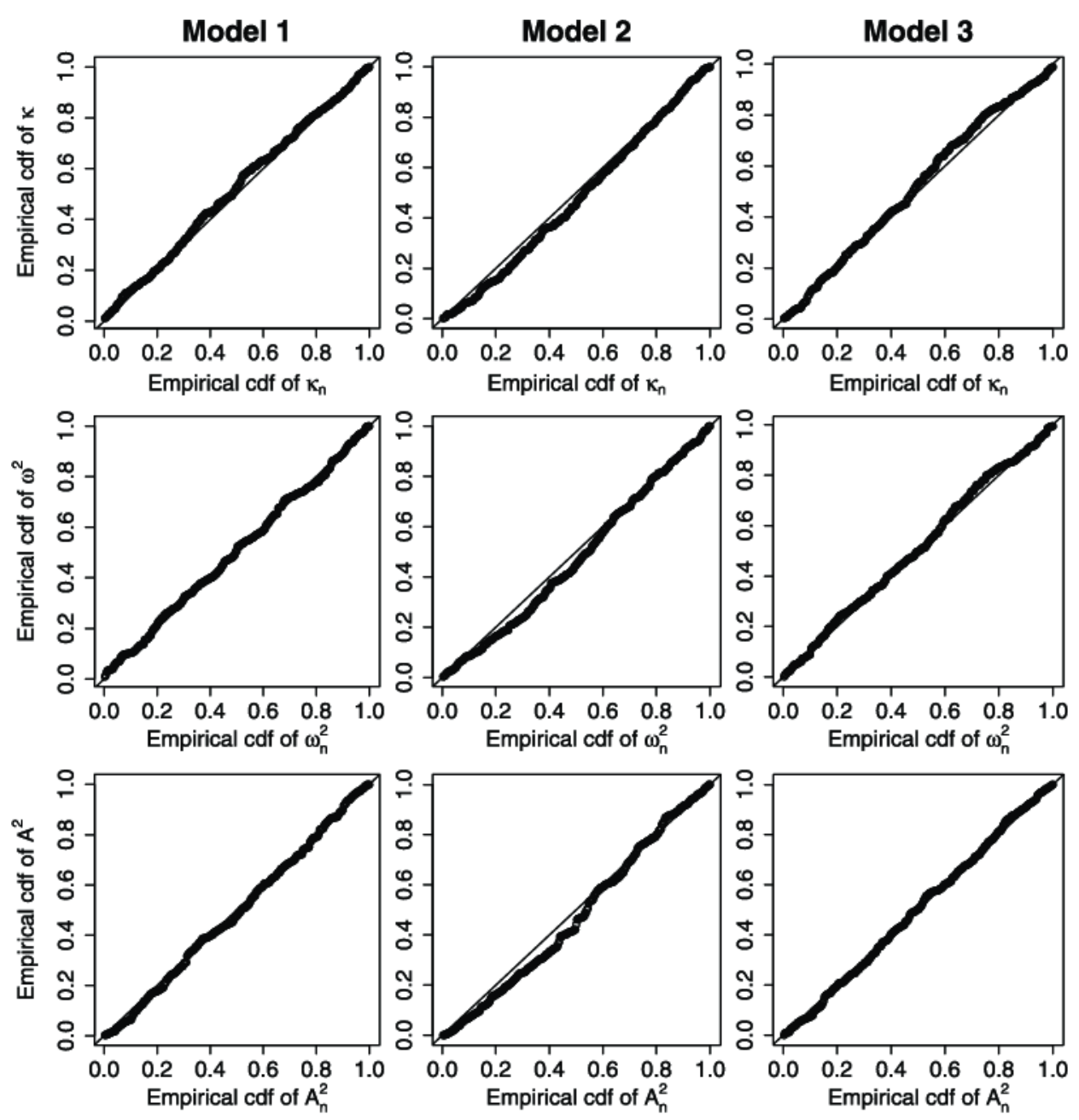

FIG. 1. PP-plots for the Kolmogorov-Smirnov, Cramér-von Mises and Anderson-Darling type test statistics.

For the 300 values of $\kappa_{n}, \omega_{n}^{2}$ and $A_{n}^{2}$ computed from the null hypothesis samples, we construct PP-plots to compare their empirical d.f.'s with the empirical d.f.'s of $\kappa, \omega^{2}$ and $A^{2}$, respectively. The results are shown in Figure 1. We see a good match of empirical d.f.'s for all three models, which shows that Theorem 4.1 yields good finite-sample approximations. This is also confirmed by the empirical size table given in the left panel of Table 1, where the observed fractions of Type I errors at the $5 \%$ significance level are shown. Note that these numbers are consistent with draws from a Binomial(300, 0.05) distribution. 
TABLE 1

Observed rejection frequencies at the 5\% significance level under null and alternative hypotheses

\begin{tabular}{rrrrrrrr}
\hline & \multicolumn{3}{c}{ Null } & & & \multicolumn{3}{c}{ Alternative } \\
\cline { 2 - 3 } & Model 1 & Model 2 & Model 3 & & Model 1 & Model 2 & Model 3 \\
\hline$\kappa_{n}$ & $15 / 300$ & $19 / 300$ & $9 / 300$ & & $97 / 100$ & $92 / 100$ & $97 / 100$ \\
$\omega_{n}^{2}$ & $16 / 300$ & $11 / 300$ & $13 / 300$ & & $99 / 100$ & $90 / 100$ & $97 / 100$ \\
$A_{n}^{2}$ & $21 / 300$ & $17 / 300$ & $18 / 300$ & & $100 / 100$ & $95 / 100$ & $100 / 100$ \\
\hline
\end{tabular}

For the 100 values of the test statistics computed under each alternative hypothesis, we present the observed fraction of rejections at the $5 \%$ significance level in the right panel of Table 1. All three tests have quite high power.

\section{Proofs.}

Proof of Theorem 3.1. First note that the terms following $V_{R}(B)$ in (24) are "annihilated" by the transformation (25):

$$
\begin{gathered}
\mathbf{Q}^{\top}(B) \mathbf{Z}-\int_{0}^{1} \mathbf{Q}^{\top}\left(B \cap A_{\mathrm{d} u}\right) \mathbf{I}^{-1}\left(A_{u}^{c}\right) \iint_{A_{u}^{c}} \mathbf{q}(x, y) \mathbf{q}^{\top}(x, y) \mathrm{d} R(x, y) \mathbf{Z} \\
=\mathbf{Q}^{\top}(B) \mathbf{Z}-\int_{0}^{1} \mathbf{Q}^{\top}\left(B \cap A_{\mathrm{d} u}\right) \mathbf{Z}=0 .
\end{gathered}
$$

Thus we can now compute, for Borel sets $B, B^{\prime} \subset[\delta, T]^{2}$,

$$
\begin{aligned}
\operatorname{Cov}[ & \left.W_{R}(B) W_{R}\left(B^{\prime}\right)\right] \\
= & E\left[V_{R}(B)-\int_{0}^{1} \mathbf{Q}^{\top}\left(B \cap A_{\mathrm{d} u}\right) \mathbf{I}^{-1}\left(A_{u}^{c}\right) \iint_{A_{u}^{c}} \mathbf{q}(x, y) \mathrm{d} V_{R}(x, y)\right] \\
& \times\left[V_{R}\left(B^{\prime}\right)-\int_{0}^{1} \mathbf{Q}^{\top}\left(B^{\prime} \cap A_{\mathrm{d} u^{\prime}}\right) \mathbf{I}^{-1}\left(A_{u^{\prime}}^{c}\right) \iint_{A_{u^{\prime}}^{c}} \mathbf{q}(x, y) \mathrm{d} V_{R}(x, y)\right] \\
= & R\left(B \cap B^{\prime}\right) \\
& -\int_{0}^{1} \mathbf{Q}^{\top}\left(B \cap A_{\mathrm{d} u}\right) \mathbf{I}^{-1}\left(A_{u}^{c}\right) \mathbf{Q}\left(B^{\prime} \cap A_{u}^{c}\right) \\
& -\int_{0}^{1} \mathbf{Q}^{\top}\left(B^{\prime} \cap A_{\mathrm{d} u^{\prime}}\right) \mathbf{I}^{-1}\left(A_{u^{\prime}}^{c}\right) \mathbf{Q}\left(B \cap A_{u^{\prime}}^{c}\right) \\
& +\int_{0}^{1} \int_{0}^{1} \mathbf{Q}^{\top}\left(B \cap A_{\mathrm{d} u}\right) \mathbf{I}^{-1}\left(A_{u}^{c}\right) \mathbf{I}\left(A_{u \vee u^{\prime}}^{c}\right) \mathbf{I}^{-1}\left(A_{u^{\prime}}^{c}\right) \mathbf{Q}\left(B^{\prime} \cap A_{\mathrm{d} u^{\prime}}\right) .
\end{aligned}
$$


Splitting the double integral into two double integrals, one over the region $\left\{u \leq u^{\prime}\right\}$ and the other over the region $\left\{u^{\prime}<u\right\}$, we see that all the integral terms cancel each other. This implies that $W_{R}$ has the covariance structure of a Wiener process with time $R$.

Let $W_{n, R}$ denote the empirical version of $W_{R}$ in Corollary 3.2,

$$
\begin{aligned}
W_{n, R}([\delta, x] \times[\delta, y]) & \\
= & \widehat{\eta}_{n}([\delta, x] \times[\delta, y]) \\
& \quad-\int_{\delta}^{x} \int_{\delta}^{y} \widehat{\mathbf{q}}^{\top}(s, t)\left(\widehat{\mathbf{I}}^{-1}(t) \int_{\delta}^{T} \int_{t}^{T} \widehat{\mathbf{q}}\left(s^{\prime}, t^{\prime}\right) \mathrm{d} \widehat{\eta}_{n}\left(s^{\prime}, t^{\prime}\right)\right) \mathrm{d} R_{\widehat{\boldsymbol{\theta}}}(s, t) .
\end{aligned}
$$

The following result will be useful for the proof of Theorem 4.1.

Proposition 7.1. Let $0<\delta<\tau<T$. If assumptions B1-B5 hold, then

$$
W_{n, R}([\delta, x] \times[\delta, y]) \Rightarrow W_{R}([\delta, x] \times[\delta, y])
$$

in $D\left([\delta, \tau]^{2}\right)$.

Proof. Applying Skorohod's representation theorem [see, e.g., Billingsley (1999), Theorem 6.7] to Theorem 2.2, we obtain a probability space that supports probabilistically equivalent versions of $\widehat{\eta}_{n}$ and $\widehat{\eta}$ satisfying

$$
\left\|\widehat{\eta}_{n}-\widehat{\eta}\right\|_{[\delta, T]^{2}} \rightarrow 0 \quad \text { a.s. }
$$

with $\|\varphi\|_{[a, b]^{2}}:=\sup _{(x, y) \in[a, b]^{2}}|\varphi(x, y)|$. We will work on this space. Let us denote

$$
\begin{gathered}
H(s, t)=\mathbf{q}^{\top}(s, t) \mathbf{I}^{-1}(t) \int_{\delta}^{T} \int_{t}^{T} \mathbf{q}\left(s^{\prime}, t^{\prime}\right) \mathrm{d} \widehat{\eta}\left(s^{\prime}, t^{\prime}\right), \\
\widehat{H}(s, t)=\widehat{\mathbf{q}}^{\top}(s, t) \widehat{\mathbf{I}}^{-1}(t) \int_{\delta}^{T} \int_{t}^{T} \widehat{\mathbf{q}}\left(s^{\prime}, t^{\prime}\right) \mathrm{d} \widehat{\eta}\left(s^{\prime}, t^{\prime}\right), \\
H_{n}(s, t)=\mathbf{q}^{\top}(s, t) \mathbf{I}^{-1}(t) \int_{\delta}^{T} \int_{t}^{T} \mathbf{q}\left(s^{\prime}, t^{\prime}\right) \mathrm{d} \widehat{\eta}_{n}\left(s^{\prime}, t^{\prime}\right), \\
\widehat{H}_{n}(s, t)=\widehat{\mathbf{q}}^{\top}(s, t) \widehat{\mathbf{I}}^{-1}(t) \int_{\delta}^{T} \int_{t}^{T} \widehat{\mathbf{q}}\left(s^{\prime}, t^{\prime}\right) \mathrm{d} \widehat{\eta}_{n}\left(s^{\prime}, t^{\prime}\right) .
\end{gathered}
$$

We have to show that

$$
\sup _{(x, y) \in[\delta, \tau]^{2}}\left|\int_{\delta}^{x} \int_{\delta}^{y}\left(\widehat{H}_{n}(s, t) r_{\widehat{\boldsymbol{\theta}}}(s, t)-H(s, t) r_{\boldsymbol{\theta}_{0}}(s, t)\right) \mathrm{d} t \mathrm{~d} s\right| \stackrel{P}{\rightarrow} 0 .
$$

For this, it suffices to prove the two statements

$$
\left\|H\left(r_{\widehat{\boldsymbol{\theta}}}-r_{\boldsymbol{\theta}_{0}}\right)\right\|_{[\delta, \tau]^{2}} \stackrel{P}{\rightarrow} 0, \quad\left\|r_{\widehat{\boldsymbol{\theta}}}\left(\widehat{H}_{n}-H\right)\right\|_{[\delta, \tau]^{2}} \stackrel{P}{\rightarrow} 0 .
$$


The first convergence in (39) follows from the continuity of $r_{\boldsymbol{\theta}}(s, t)$ over $(s, t, \boldsymbol{\theta}) \in[\delta, T]^{2} \times B\left(\boldsymbol{\theta}_{0}\right)$ and the continuity of $H(s, t)$ over $(s, t) \in[\delta, \tau]^{2}$. The second convergence in (39) follows from

$$
\left\|\widehat{H}_{n}-H\right\|_{[\delta, \tau]^{2}} \stackrel{P}{\rightarrow} 0,
$$

since $\left\|r_{\widehat{\boldsymbol{\theta}}}\right\|_{[\delta, \tau]^{2}}=O_{P}(1)$. We establish (40) by proving the two statements

$$
\left\|\widehat{H}_{n}-H_{n}\right\|_{[\delta, \tau]^{2}} \stackrel{P}{\rightarrow} 0, \quad\left\|H_{n}-H\right\|_{[\delta, \tau]^{2}} \stackrel{P}{\rightarrow} 0 .
$$

Consider the second statement in (41). Its left-hand side is equal to

$$
\sup _{(s, t) \in[\delta, \tau]^{2}}\left|\mathbf{q}^{\top}(s, t) \mathbf{I}^{-1}(t) \int_{\delta}^{T} \int_{t}^{T} \mathbf{q}\left(s^{\prime}, t^{\prime}\right) \mathrm{d} \Delta_{n}\left(s^{\prime}, t^{\prime}\right)\right|,
$$

with $\Delta_{n}=\widehat{\eta}_{n}-\widehat{\eta}$. The vector function $\mathbf{q}^{\top}(s, t) \mathbf{I}^{-1}(t)$ is bounded on $(s, t) \in$ $[\delta, \tau]^{2}$, by continuity. So it will suffice to show

$$
\sup _{t \in[\delta, \tau]}\left|\int_{\delta}^{T} \int_{t}^{T} q_{i}\left(s^{\prime}, t^{\prime}\right) \mathrm{d} \Delta_{n}\left(s^{\prime}, t^{\prime}\right)\right| \stackrel{P}{\rightarrow} 0, \quad i=1, \ldots, 6+d .
$$

The double integral inside the absolute value bars can be rewritten, using integration by parts [see Hildebrandt (1963), Section III.8], as follows:

$$
\begin{aligned}
q_{i}(T, & T) \Delta_{n}(T, T)-q_{i}(T, t) \Delta_{n}(T, t)-q_{i}(\delta, T) \Delta_{n}(\delta, T)+q_{i}(\delta, t) \Delta_{n}(\delta, t) \\
& -\int_{\delta}^{T} \Delta_{n}\left(s^{\prime}, T\right) \mathrm{d} q_{i}\left(s^{\prime}, T\right)+\int_{\delta}^{T} \Delta_{n}\left(s^{\prime}, t\right) \mathrm{d} q_{i}\left(s^{\prime}, t\right) \\
& -\int_{t}^{T} \Delta_{n}\left(T, t^{\prime}\right) \mathrm{d} q_{i}\left(T, t^{\prime}\right)+\int_{t}^{T} \Delta_{n}\left(\delta, t^{\prime}\right) \mathrm{d} q_{i}\left(\delta, t^{\prime}\right) \\
& +\int_{\delta}^{T} \int_{t}^{T} \Delta_{n}\left(s^{\prime}, t^{\prime}\right) \mathrm{d} q_{i}\left(s^{\prime}, t^{\prime}\right) .
\end{aligned}
$$

Each of the first four terms is bounded in absolute value by $\left\|q_{i}\right\|_{[\delta, T]^{2}}$. $\left\|\Delta_{n}\right\|_{[\delta, T]^{2}}$, where the first factor is finite by continuity and the second factor vanishes in probability. Moreover, each integral term is bounded in absolute value by $\left\|q_{i}\right\|_{\mathrm{HK}}\left\|\Delta_{n}\right\|_{[\delta, T]^{2}}$, which also vanishes in probability because $\left\|q_{i}\right\|_{\mathrm{HK}}<\infty$, by virtue of the assumptions $\left\|\rho_{j}\left(x, y, \boldsymbol{\theta}_{0}\right)\right\|_{\mathrm{HK}}<\infty$ for $j=1, \ldots, 2+d$, and Proposition 1 of Blümlinger and Tichy (1989). Hence (42) follows, and the second convergence in (41) is established.

It remains to prove the first convergence in (41). By virtue of the second convergence there, and an analogous result for $\widehat{H}_{n}$ and $\widehat{H}$, it will suffice to prove $\|\widehat{H}-H\|_{[\delta, \tau]^{2}} \stackrel{P}{\rightarrow} 0$. Note that

$$
|\widehat{H}(s, t)-H(s, t)|
$$




$$
\begin{aligned}
\leq & \left|\widehat{\mathbf{q}}^{\top}(s, t) \widehat{\mathbf{I}}^{-1}(t)-\mathbf{q}^{\top}(s, t) \mathbf{I}^{-1}(t)\right| \cdot\left|\int_{\delta}^{T} \int_{t}^{T} \mathbf{q}\left(s^{\prime}, t^{\prime}\right) \mathrm{d} \widehat{\eta}\left(s^{\prime}, t^{\prime}\right)\right| \\
& +\left|\widehat{\mathbf{q}}^{\top}(s, t) \widehat{\mathbf{I}}^{-1}(t)\right| \cdot\left|\int_{\delta}^{T} \int_{t}^{T}\left(\widehat{\mathbf{q}}\left(s^{\prime}, t^{\prime}\right)-\mathbf{q}\left(s^{\prime}, t^{\prime}\right)\right) \mathrm{d} \widehat{\eta}\left(s^{\prime}, t^{\prime}\right)\right|,
\end{aligned}
$$

where $|\cdot|$ should be interpreted component-wise.

Let us write $\mathbf{q}\left(s, t, z_{1}, z_{2}, w_{1}, \ldots, w_{d}\right)$ to denote the vector $\mathbf{q}(s, t)$ with the values of $\gamma_{1}$ and $\gamma_{2}$ replaced by variables $z_{1}$ and $z_{2}$, and the values $\theta_{01}, \ldots, \theta_{0 d}$ replaced by variables $w_{1}, \ldots, w_{d}$. Then $\mathbf{q}(s, t)=\mathbf{q}\left(s, t, \gamma_{1}, \gamma_{2}, \theta_{01}, \ldots, \theta_{0 d}\right)$ and $\widehat{\mathbf{q}}(s, t)=\mathbf{q}\left(s, t, \widehat{\gamma}_{1}, \widehat{\gamma}_{2}, \widehat{\theta}_{1}, \ldots, \widehat{\theta}_{d}\right)$.

Now consider the first term on the right-hand side of (43). Since the vector $\mathbf{q}\left(s, t, z_{1}, z_{2}, w_{1}, \ldots, w_{d}\right)$ is continuous over $[\delta, \tau]^{2} \times \mathbb{R}^{2} \times B\left(\boldsymbol{\theta}_{0}\right)$, we have that $\left|\widehat{\mathbf{q}}^{\top}(s, t) \widehat{\mathbf{I}}^{-1}(t)-\mathbf{q}^{\top}(s, t) \mathbf{I}^{-1}(t)\right|$ is $o_{P}(1)$ uniformly over $(s, t) \in$ $[\delta, \tau]^{2}$. Moreover, an integration by parts argument as above yields that

$$
\left|\int_{\delta}^{T} \int_{t}^{T} q_{i}\left(s^{\prime}, t^{\prime}\right) \mathrm{d} \widehat{\eta}\left(s^{\prime}, t^{\prime}\right)\right| \leq\|\widehat{\eta}\|_{[\delta, T]^{2}} \cdot\left(4\left\|q_{i}\right\|_{[\delta, T]^{2}}+5\left\|q_{i}\right\|_{\mathrm{HK}}\right)
$$

for $1 \leq i \leq 6+d$, where the right-hand side is $O_{P}(1)$. We conclude that the first term on the right-hand side of (43) is $o_{P}(1)$ uniformly over $(s, t) \in[\delta, \tau]^{2}$.

Next, consider the second term on the right-hand side of (43). It follows from the discussion above that the vector $\left|\widehat{\mathbf{q}}^{\top}(s, t) \widehat{\mathbf{I}}^{-1}(t)\right|$ is $O_{P}(1)$ uniformly over $(s, t) \in[\delta, \tau]^{2}$, so it will suffice to show that

$$
\sup _{t \in[\delta, \tau]}\left|\int_{\delta}^{T} \int_{t}^{T} \Delta q_{i}\left(s^{\prime}, t^{\prime}\right) \mathrm{d} \widehat{\eta}\left(s^{\prime}, t^{\prime}\right)\right| \stackrel{P}{\rightarrow} 0,
$$

with $\Delta q_{i}=\widehat{q}_{i}-q_{i}$, for $i=1, \ldots, 6+d$. Once again, an integration by parts argument shows that the left-hand side of (44) is bounded from above by

$$
\|\widehat{\eta}\|_{[\delta, T]^{2}} \cdot\left(4\left\|\Delta q_{i}\right\|_{[\delta, T]^{2}}+5\left\|\Delta q_{i}\right\|_{\mathrm{HK}}\right),
$$

where $\|\widehat{\eta}\|_{[\delta, T]^{2}}<\infty$ a.s. and $\left\|\Delta q_{i}\right\|_{[\delta, T]^{2}}=o_{P}(1)$ by continuity. It remains to establish $\left\|\Delta q_{i}\right\|_{\mathrm{HK}}=o_{P}(1)$. For $i=7, \ldots, 6+d$, this follows directly from assumption B5. For $i=1$, we have

$$
\begin{aligned}
\left\|\Delta q_{1}\right\|_{\mathrm{HK}} & =\left\|f_{1}\left(x, \widehat{\gamma}_{1}\right) \rho_{1}(x, y, \widehat{\boldsymbol{\theta}})-f_{1}\left(x, \gamma_{1}\right) \rho_{1}\left(x, y, \boldsymbol{\theta}_{0}\right)+\Delta f_{1}^{\prime}(x)\right\|_{\mathrm{HK}} \\
& \leq\left\|\Delta f_{1}(x) \rho_{1}\left(x, y, \boldsymbol{\theta}_{0}\right)\right\|_{\mathrm{HK}}+\left\|f_{1}\left(x, \widehat{\gamma}_{1}\right) \Delta \rho_{1}(x, y)\right\|_{\mathrm{HK}}+2 V^{(1)}\left(\Delta f_{1}^{\prime}\right) .
\end{aligned}
$$

Using Proposition 1 of Blümlinger and Tichy (1989), differentiability properties of $f_{1}, f_{1}^{\prime}$ on $[\delta, T]$ and assumption B5, each term on the right-hand side can be shown to be $o_{P}(1)$. The cases $i=2, \ldots, 6$ are similar. Thus (44) follows. 
Proof of Theorem 4.1. Note that we have

$$
\begin{aligned}
W_{n}([\delta, x] \times[\delta, y]) & =\int_{\delta}^{x} \int_{\delta}^{y} \sigma(s, t, \widehat{\boldsymbol{\theta}}) \mathrm{d} W_{n, R}([\delta, s] \times[\delta, t]), \\
W([\delta, x] \times[\delta, y]) & =\int_{\delta}^{x} \int_{\delta}^{y} \sigma\left(s, t, \boldsymbol{\theta}_{0}\right) \mathrm{d} W_{R}([\delta, s] \times[\delta, t]) .
\end{aligned}
$$

Now, by Proposition 7.1 and Skorohod's representation theorem, there exists a probability space supporting versions of $W_{n, R}$ and $W_{R}$ which satisfy

$$
\begin{gathered}
\sup _{(x, y) \in[\delta, \tau]^{2}}\left|W_{n, R}([\delta, x] \times[\delta, y])-W_{R}([\delta, x] \times[\delta, y])\right| \\
=: \sup _{(x, y) \in[\delta, \tau]^{2}}\left|D_{n}(x, y)\right| \rightarrow 0 \quad \text { a.s. }
\end{gathered}
$$

We work with this probability space. We have

$$
\begin{aligned}
& \left|W_{n}([\delta, x] \times[\delta, y])-W([\delta, x] \times[\delta, y])\right| \\
& \quad \leq\left|\int_{\delta}^{x} \int_{\delta}^{y} \Delta \sigma(s, t) \mathrm{d} W_{R}([\delta, s] \times[\delta, t])\right|+\left|\int_{\delta}^{x} \int_{\delta}^{y} \sigma(s, t, \widehat{\boldsymbol{\theta}}) \mathrm{d} D_{n}(s, t)\right| .
\end{aligned}
$$

Applying integration by parts as in the proof of Proposition 7.1, we see that the first term on the right-hand side of (45) is bounded by

$$
\sup _{(s, t) \in[\delta, \tau]^{2}}\left|W_{R}([\delta, s] \times[\delta, t])\right| \cdot\left(4\|\Delta \sigma\|_{[\delta, \tau]^{2}}+5\|\Delta \sigma\|_{\mathrm{HK}}\right) .
$$

Since $W_{R}$ is a.s. bounded on $[\delta, \tau]^{2},\|\Delta \sigma\|_{[\delta, \tau]^{2}}=o_{P}(1)$ by continuity, and $\|\Delta \sigma\|_{\mathrm{HK}}=o_{P}(1)$ by assumption B5, (46) vanishes in probability. Similarly, the second term on the right-hand side of (45) is bounded by

$$
\left\|D_{n}\right\|_{[\delta, \tau]^{2}} \cdot\left(4\|\sigma(\cdot, \cdot, \widehat{\boldsymbol{\theta}})\|_{[\delta, \tau]^{2}}+5\|\sigma(\cdot, \cdot, \widehat{\boldsymbol{\theta}})\|_{\mathrm{HK}}\right),
$$

which also vanishes in probability since $\left\|D_{n}\right\|_{[\delta, \tau]^{2}}=o_{P}(1)$ and the two summands in the parentheses are $O_{P}(1)$. Thus the left-hand side of (45) is $o_{P}(1)$ uniformly over $(s, t) \in[\delta, \tau]^{2}$.

Acknowledgements. We are very grateful to an Associate Editor and three referees for many insightful comments and suggestions that led to this improved version of the manuscript. We are also grateful to the participants of the 2013 Extreme Value Analysis conference in Shanghai and the Fourth Wellington Workshop in Probability and Mathematical Statistics (WWPMS4) for their feedback.

\section{SUPPLEMENTARY MATERIAL}

Supplement to "Asymptotically distribution-free goodness-of-fit testing for tail copulas" (DOI: 10.1214/14-AOS1304SUPP; .pdf). We provide a proof of Theorem 2.1 as well as details about the Monte Carlo simulations of Section 6. 


\section{REFERENCES}

Beirlant, J., Goegebeur, Y., Teugels, J. and Segers, J. (2004). Statistics of Extremes: Theory and Applications. Wiley, Chichester. MR2108013

Billingsley, P. (1999). Convergence of Probability Measures, 2nd ed. Wiley, New York. MR1700749

Blümlinger, M. and Tichy, R. F. (1989). Topological algebras of functions of bounded variation. I. Manuscripta Math. 65 245-255. MR1011435

Can, S., Einmahl, J. J., Khmaladze, E. V. and Laeven, R. A. (2015). Supplement to "Asymptotically distribution-free goodness-of-fit testing for tail copulas." DOI:10.1214/14-AOS1304SUPP.

Coles, S. G. and TAwn, J. A. (1991). Modelling extreme multivariate events. J. Roy. Statist. Soc. Ser. B 53 377-392. MR1108334

DE HAAn, L. and FerReIrA, A. (2006). Extreme Value Theory: An Introduction. Springer, New York. MR2234156

De HaAn, L., Neves, C. and Peng, L. (2008). Parametric tail copula estimation and model testing. J. Multivariate Anal. 99 1260-1275. MR2419346

DE HAAn, L. and Resnick, S. I. (1977). Limit theory for multivariate sample extremes. Z. Wahrsch. Verw. Gebiete 40 317-337. MR0478290

DE HAAN, L. and RESNICK, S. I. (1993). Estimating the limit distribution of multivariate extremes. Comm. Statist. Stochastic Models 9 275-309. MR1213072

Delgado, M. A., Hidalgo, J. and Velasco, C. (2005). Distribution free goodness-of-fit tests for linear processes. Ann. Statist. 33 2568-2609. MR2253096

Dette, H. and Hetzler, B. (2009). Khmaladze transformation of integrated variance processes with applications to goodness-of-fit testing. Math. Methods Statist. 18 97-116. MR2537360

EINMAhL, J. H. J., DE HAAN, L. and LI, D. (2006). Weighted approximations of tail copula processes with application to testing the bivariate extreme value condition. Ann. Statist. 34 1987-2014. MR2283724

Einmahl, J. H. J., DE HAAn, L. and Sinha, A. K. (1997). Estimating the spectral measure of an extreme value distribution. Stochastic Process. Appl. 70 143-171. MR1475660

Einmahl, J. H. J., Krajina, A. and Segers, J. (2008). A method of moments estimator of tail dependence. Bernoulli 14 1003-1026. MR2543584

Einmahl, J. H. J., Krajina, A. and Segers, J. (2012). An $M$-estimator for tail dependence in arbitrary dimensions. Ann. Statist. 40 1764-1793. MR3015043

HARDY, G. H. (1905). On double Fourier series, and especially those which represent the double zeta-function with real and incommensurable parameters. Quart. J. Math. 37 $53-79$.

Hildebrandt, T. H. (1963). Introduction to the Theory of Integration. Academic Press, New York. MR0154957

Huang, X. (1992). Statistics of bivariate extreme values. Ph.D. thesis, Univ. Rotterdam.

Joe, H., Smith, R. L. and Weissman, I. (1992). Bivariate threshold methods for extremes. J. Roy. Statist. Soc. Ser. B 54 171-183. MR1157718

Khmaladze, E. V. (1981). A martingale approach in the theory of goodness-of-fit tests. Teor. Veroyatnost. i Primenen. 26 246-265. MR0616619

Khmaladze, E. V. (1988). An innovation approach to goodness-of-fit tests in $\mathbf{R}^{m}$. Ann. Statist. 16 1503-1516. MR0964936

Khmaladze, E. V. (1993). Goodness of fit problem and scanning innovation martingales. Ann. Statist. 21 798-829. MR1232520

Khmaladze, E. V. and Koul, H. L. (2004). Martingale transforms goodness-of-fit tests in regression models. Ann. Statist. 32 995-1034. MR2065196 
Khmaladze, E. V. and Koul, H. L. (2009). Goodness-of-fit problem for errors in nonparametric regression: Distribution free approach. Ann. Statist. 37 3165-3185. MR2549556

Koenker, R. and XiaO, Z. (2002). Inference on the quantile regression process. Econometrica 70 1583-1612. MR1929979

Koenker, R. and Xiao, Z. (2006). Quantile autoregression. J. Amer. Statist. Assoc. 101 980-990. MR2324109

Kotz, S. and Nadarajah, S. (2000). Extreme Value Distributions: Theory and Applications. Imperial College Press, London. MR1892574

Koul, H. L. and Swordson, E. (2011). Khmaladze transformation. In International Encyclopedia of Statistical Science (M. LovrIc, ed.) 715-718. Springer, Berlin.

Krause, M. (1903). Fouriersche Reihen mit zwei veränderlichen Grössen. Ber. Sächs. Akad. Wiss. Leipzig 55 164-197.

McKeague, I. W., Nikabadze, A. M. and Sun, Y. Q. (1995). An omnibus test for independence of a survival time from a covariate. Ann. Statist. 23 450-475. MR1332576

NikABAdZe, A. and Stute, W. (1997). Model checks under random censorship. Statist. Probab. Lett. 32 249-259. MR1440835

Owen, A. B. (2005). Multidimensional variation for quasi-Monte Carlo. In Contemporary Multivariate Analysis and Design of Experiments. Ser. Biostat. 2 49-74. World Sci. Publ., Hackensack, NJ. MR2271076

Stute, W., Thies, S. and Zhu, L.-X. (1998). Model checks for regression: An innovation process approach. Ann. Statist. 26 1916-1934. MR1673284

S. U. CAN

R. J. A. LAEven

FACUlty of ECONOMics \& Business

Section Actuarial Science

UNIVERSITY OF AMSTERDAM

VALCKENIERSTRAAT 65

1018 XE AMSTERDAM

The Netherlands

E-MAIL: s.u.can@uva.nl

$$
\text { r.j.a.laeven@uva.nl }
$$

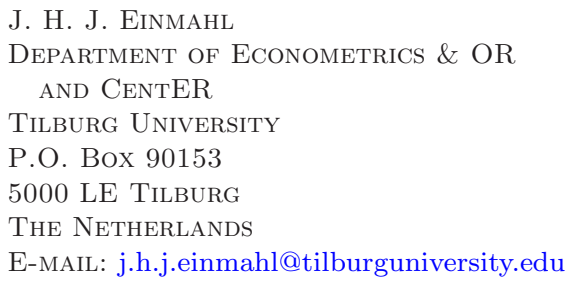

E. V. KhmaladZe

School of Mathematics, Statistics \& OR

ViCTORIA UNIVERSITY OF WeLLingtoN

P.O. Box 600

Wellington

NEW ZEALAND

E-MAIL: estate.khmaladze@vuw.ac.nz 